\title{
Towards Restoring Locomotion for Paraplegics: Realizing Dynamically Stable Walking on Exoskeletons
}

\author{
Thomas Gurriet $^{1}$, Sylvain Finet ${ }^{2}$, Guilhem Boeris ${ }^{2}$, Alexis Duburcq ${ }^{2}$, Ayonga Hereid ${ }^{3}$, Omar Harib ${ }^{3}$, \\ Matthieu Masselin ${ }^{2}$, Jessy Grizzle ${ }^{3}$ and Aaron D. Ames ${ }^{1}$
}

\begin{abstract}
This paper presents the first experimental results of crutch-less dynamic walking with paraplegics on a lowerbody exoskeleton: ATALANTE, designed by the French start-up company Wandercraft. The methodology used to achieve these results is based on the partial hybrid zero dynamics (PHZD) framework for formally generating stable walking gaits. A direct collocation optimization formulation is used to provide fast and efficient generation of gaits tailored to each patient. These gaits are then implemented on the exoskeleton for three paraplegics. The end result is dynamically stable walking in an exoskeleton without the need for crutches. After a short period of tuning by the engineers and practice by the subjects, each subject was able to dynamically walk across a room of about $10 \mathrm{~m}$ up to a speed of $0.15 \mathrm{~m} / \mathrm{s}(0.5 \mathrm{~km} / \mathrm{h})$ without the need for crutches or any other kind of assistance.
\end{abstract}

\section{INTRODUCTION}

Regaining autonomy in their daily lives through the ability to walk again is a central aspiration for many people with lower limb paralysis, i.e. paraplegics. Among the promising solutions to restore locomotion are robotic devices that fit around the legs of a user, called lower-limb exoskeletons for rehabilitation. A comprehensive review of the state-of-theart lower-limb exoskeletons can be found in [10]. Research in this field began in the early 1960s independently at the Mihailo Pupin Institute in Belgrade [18], [26] and at the University of Wisconsin [15], [24]. The resulting exoskeletons were able to physically advance the legs of a user, but the user had to use crutches to maintain balance. Additionally, there has been a broad body of work on different aspect of exoskeletons [10], [25], [29] from assistive knee devices [11] to soft exoskeletons [27]. Throughout the years, in addition to regaining autonomy, clinical tests showed the health benefits of walking for paraplegics: improvement of blood circulation, of respiratory, urinary, and intestinal functions, and positive psychological effects [20].

Due to the physical and psychological advantages of exoskeletons, multiple companies have emerged over the past decade and have started to commercialize their products for

\footnotetext{
*This work has been conducted under IRB No. 16-0693.

${ }^{1}$ Thomas Gurriet and Aaron Ames are with the Department of Mechanical and Civil Engineering, California Institute of Technology, Pasadena, CA 91125. Their work was supported by NSF NRI award 1526519.

${ }^{2}$ Matthieu Masselin, Sylvain Finet, Guilhem Boeris and Alexis Duburcq are with Wandercraft, Paris, France.

${ }^{3}$ Ayonga Hereid, Omar Harib and Jessy Grizzle are with the Department of Electrical Engineering and Computer Science, Univ. of Michigan, Ann Arbor, MI 48109. The work of A. Hereid was supported by NSF under Grant CPS-1239037. The work of J. W. Grizzle was partially supported by a gift from Ford Motor Company.
}

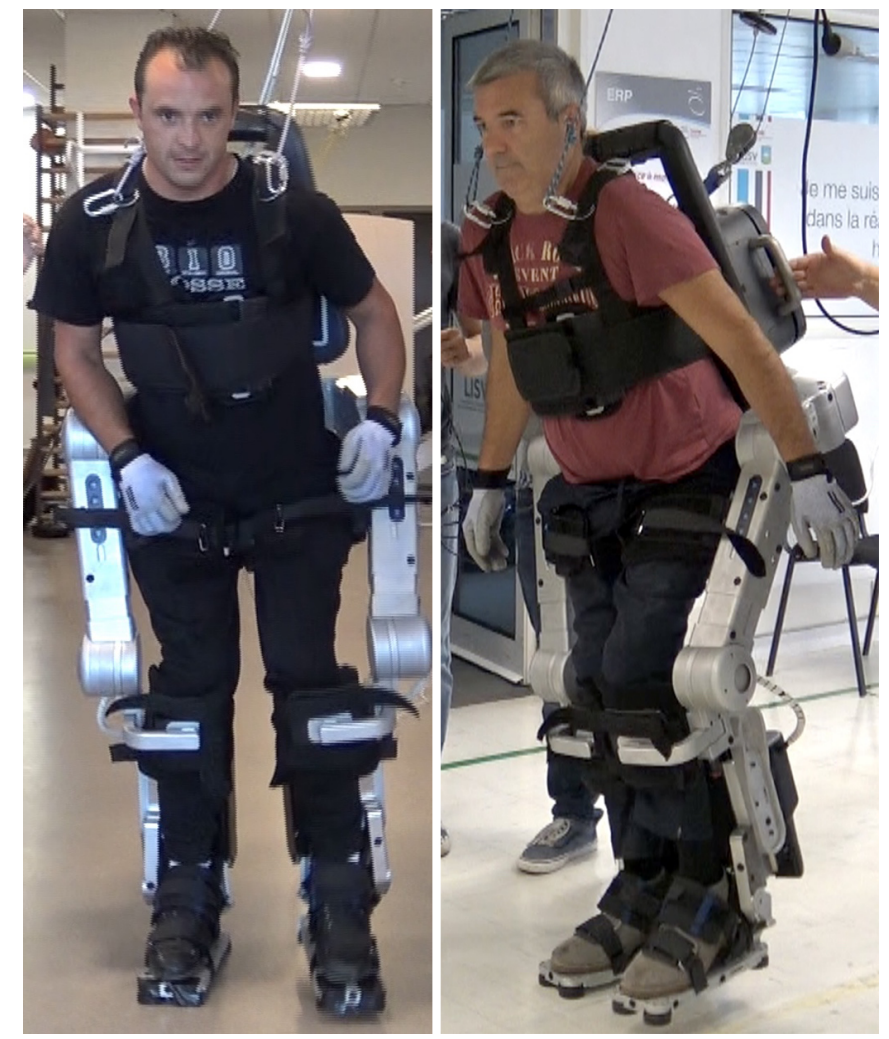

Fig. 1: Image of two paraplegic patients walking in the ATALANTE exoskeleton developed by Wandercraft.

use in rehabilitation centers. Nevertheless, these exoskeletons still have very limited capabilities: either they require the use of crutches for balance or direction control, which is tiring for the user [1], [2], or they provide slow static gaits with velocities on the order of $0.05 \mathrm{~m} / \mathrm{s}$ [3]. The ultimate goal is to have a device that provides fast, robust and dynamic gaits for safe daily use anywhere. By "dynamic", as opposed to "static", we mean that the high level control goal is to stabilize periodic motions that do not necessarily keep the center of mass (or even of center of pressure) within a support polygon of the feet. From an outside perspective, during a step the exoskeleton is falling forward only to have the swing foot reach out and catch the user before beginning the process again. This is the behavior naturally achieved by humans when they walk, and the objective is thus to endow exoskeletons with these behaviors. The French start-up company Wandercraft has emerged with the goal of designing such devices [5]. 


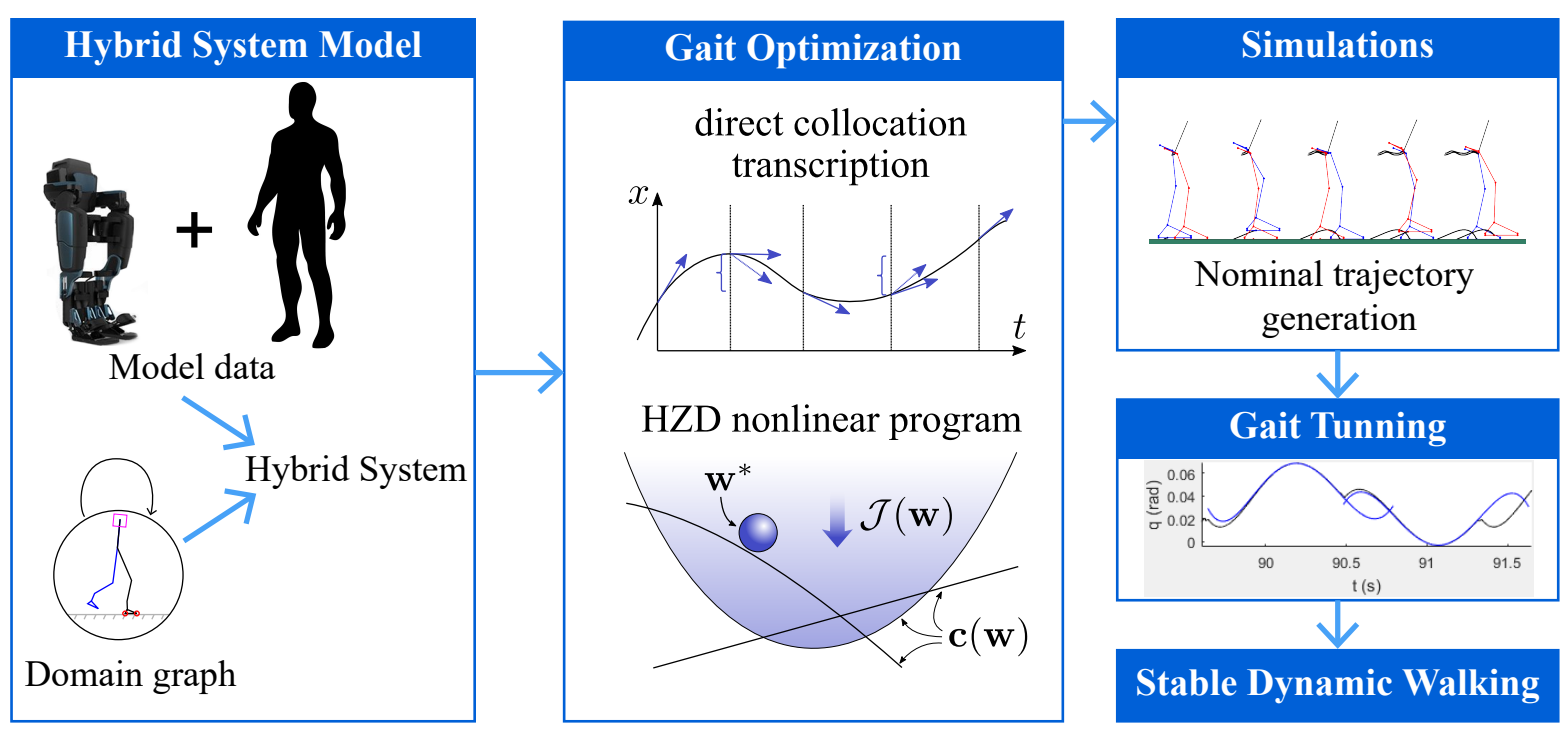

Fig. 2: The methodology developed that yielded dynamically stable exoskeleton walking experimentally for paraplegics.

Recent progress in the field of robotic bipedal locomotion, both in terms of hardware development and control algorithm design, is making dynamic locomotion on exoskeletons a possibility. Of particular note for this work is the the method of virtual constraints and hybrid zero dynamics (HZD method for short) which has resulted in dynamic walking on multiple robots by facilitating the design of gaits that are provably stable and experimentally realizable; they enforce the unilateral ground contact as well as friction and input torque bounds [8], [12], [13], [17], [28]. Virtual constraints are relations between the joints or links of a device that are induced by feedback control instead of a physical connection. They thus induce a reduced-order dynamic model of the hybrid system that is called the hybrid zero dynamics. The method of HZD was extended to allow for multi-contact walking for robots with feet in the context of partial hybrid zero dynamics, or PHZD for short [7]. PHZD has been successfully realized to achieve dynamic and efficient locomotion on humanoid robots with feet [17], [22], [23] and, as a result, is a natural framework in which to control exoskeletons with the goal of dynamic and human-like gaits. Quite recently, preliminary simulation results showed the application of PHZD to the control of an exoskeleton worn by a paraplegic; see [6] for details as well as later in the paper.

Wandercraft has designed an exoskeleton, named ATALANTE, which has realized for the first time, using controllers based on the PHZD method, dynamic stable walking for paraplegics without the use of crutches or any other kind of assistance (as illustrated in Fig. 1). This paper describes the formal foundations along with the methodology used for achieving this performance and presents some promising experimental results: after a short period of tuning by onsite engineers and practice by the subjects, each subject was able to dynamically walk across a room of about $10 \mathrm{~m}$ up to a speed of $0.15 \mathrm{~m} / \mathrm{s}(0.5 \mathrm{~km} / \mathrm{h})$. Although it reports results obtained with patients in clinical settings, it is not a clinical report of any sort. Therefore, the contributions of this paper are:

- The application of the PHZD method to the experimental control of a lower-limb exoskeleton.

- The realization of dynamically stable walking for paraplegics without the use of crutches or any other kind of assistance.

The remainder of the paper is organized as follows (following the methodology in Fig. 2). Section II presents a hybrid model of the human exoskeleton system and the PHZD framework. This leads to our framework for the design of stable dynamic walking gaits represented as a nonlinear optimization problem under constraints. The resolution of this problem using direct collocation is described in Section III. Finally, Section IV presents the implementation of the gaits on the exoskeleton and the experimental results.

\section{Mathematical Foundation}

In this section, we summarize our approach for generating stabilizing controllers for the powered lower-limb exoskeleton ATALANTE by building upon the (PHZD)based theoretical foundation [7]. More precisely, we utilize the hybrid nature of bipedal locomotion and implement stable periodic behaviors by enforcing invariant reduced dimensional manifolds via virtual-constraint-based feedback controllers. Because in these experiments, the user is not yet able to use his or her upper body to select the speed of the gait, the presentation does not require the decentralized implementation presented in [6].

\section{A. Hybrid System Model}

ATALANTE is a lower-limb exoskeleton designed by the French startup company Wandercraft. It is intended to be used by paraplegics in medical center settings for rehabilitation. The exoskeleton has 12 degrees of freedom (6 per leg). Except for the ankle, where a special mechanism is mounted, 


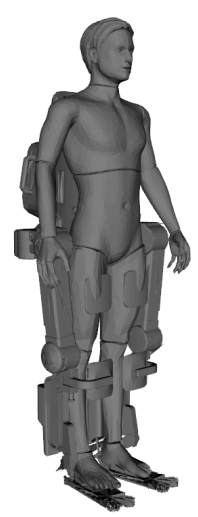

(a) Human Exoskeleton Model

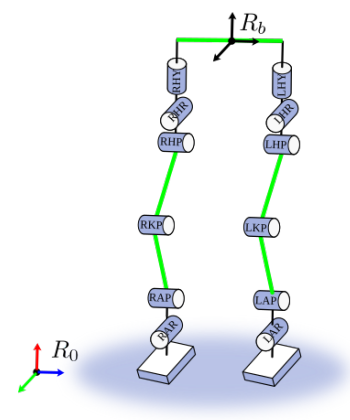

(b) Kinematic Tree
Fig. 3: Kinematic diagram of the human exoskeleton system. The green links in (b) are adjustable in length.

each degree of freedom is independently actuated by a brushless DC motor. A digital encoder is mounted on each motor to estimate joint position and velocity. Sensors installed in the feet allow for impact detection when the feet hit the ground. The exoskeleton is controlled by a central computer board running a real-time operating system and in charge of all high-level computations. The dimensions of ATALANTE can be easily adjusted to fit the patient through mechanical adjustments. The patient is secured to the exoskeleton by means of fasteners located at the ankle, the shin, the thigh, and the abdomen. ATALANTE is self-powered with a battery pack.

One key assumption in the current paper ${ }^{1}$ is that the user's legs are rigidly attached to the exoskeleton and there is no actuation from the human body. The upper human body is modeled as a single rigid link-ignoring the relative motion of the waist, neck and arm joints of the human-rigidly attached to the pelvis of the exoskeleton. The lumped humanexoskeleton system then can be modeled as a kinematic tree of rigid links, as shown in Fig. 3. Specifically, we use floating base coordinates: assuming $R_{b}$ is a body-fixed reference frame of the pelvis link with Cartesian position $p \in \mathbb{R}^{3}$ and orientation $\phi \in S O(3)$ with respect to the world frame $R_{0}$, the configuration coordinates, $\mathscr{Q}$, of the system, given as

$$
q=\left(p, \phi, q_{b}\right) \in \mathscr{Q}=\mathbb{R}^{3} \times S O(3) \times \mathscr{Q}_{b},
$$

represents the generalized coordinates of the system. The body coordinates, $q_{b} \in \mathscr{Q}_{b}$, represent the 12 actuated lowerlimb exoskeleton joints-each leg consists of 3 hip joints, 1 knee joint, and 2 ankle joints.

The dynamic behavior of bipedal locomotion is often characterized as a hybrid system consisting of a series of continuous phases (e.g., when the leg swings forward) and discrete events (e.g. when the foot strikes the ground) [14]. In particular, we model the walking behavior of the human

\footnotetext{
${ }^{1}$ In [6] we allow the user to control the exoskeleton via upper body posture; hence an articulated model of a human is used.
}

exoskeleton system as a hybrid control system (see [6] for full definition):

$$
\mathscr{H} \mathscr{C}=\{\mathscr{D}, \mathscr{U}, S, \Delta, F G\},
$$

where $\mathscr{D} \subseteq T \mathscr{Q}$ is a smooth manifold representing the admissible domain of the states, $\mathscr{U}$ is the admissible control inputs, $S \subset \mathscr{D}$ is a switching surface, $\Delta$ is the reset map of the discontinuous dynamics, and $F G$ is the control system defined on the continuous domain. The definition of each element of (2) is discussed briefly below.

Continuous Dynamics. For flat-footed walking, the continuous domain $\mathscr{D}$ is determined by the planar contact of the stance foot with the ground. Assuming the ground is rigid, we will use holonomic constraints to model the contact, i.e., the position and the orientation of the stance foot link remain constant during the course of one step. Let $\mu(q) \in \mathbb{R}^{6}$ be the collection of stance foot position and orientation, the kinematic constraint of the foot contact is $J(q) \dot{q}=0$, where $J(q)=\frac{\partial \mu(q)}{\partial q}$ is the Jacobian of holonomic constraints.

Given the mass, inertia, and length properties of each link of the human exoskeleton system, the equation of motion (EOM) of the continuous dynamics is then determined by the classical Euler-Lagrange equation and holonomic constraints of the domain [14], [21]:

$$
D(q) \ddot{q}+H(q, \dot{q})=B u+J^{T}(q) F
$$

where $D(q)$ is the inertia matrix, $H(q, \dot{q})=C(q, \dot{q}) \dot{q}+G(q)$ is the vector containing the Coriolis and gravity term, $B$ is the actuator distribution matrix, $u \in \mathscr{U} \subseteq \mathbb{R}^{12}$ is the actuator inputs vector. The contact wrenches, $F$, can be calculated by solving the second order derivative of the holonomic constraints,

$$
J(q) \ddot{q}+\dot{J}(q, \dot{q}) \dot{q}=0,
$$

and (3) simultaneously. Substituting the closed form solution of $F$ yields the affine control system, $F G$, given as,

$$
\dot{x}=f(x)+g(x) u,
$$

with $x=(q, \dot{q}) \in \mathscr{D}$ being the state of the system.

Discrete Dynamics. When the swing foot hits the ground, an instantaneous change in contact occurs. We denote the triggering condition of the event as a guard $S \subset \mathscr{D}$. Moreover, we assume that the impact with the ground is perfectly plastic. As a consequence, the joint configuration remains the same during impact, but the joint velocities undergo a discrete change. More precisely, using the derivation proposed by Hurmuzlu et al. [19], we get:

$$
\dot{q}^{+}=\left(I-D^{-1}\left(q^{+}\right) J^{T}\left(q^{+}\right) \Xi\left(q^{+}\right) J\left(q^{+}\right)\right) \dot{q}^{-}:=\Delta_{\dot{q}}(q) \dot{q}^{-},
$$

where, for simplicity, $\Xi(q)=\left(J(q) D^{-1}(q) J^{T}(q)\right)^{-1}$. This discrete dynamics of the system can be captured as a reset map, $\Delta$, that maps the pre-impact states to post-impact states:

$$
\left(q^{+}, \dot{q}^{+}\right)=\Delta\left(q^{-}, \dot{q}^{-}\right):=\left[\begin{array}{c}
q^{-} \\
\Delta_{\dot{q}}(q) \dot{q}^{-}
\end{array}\right] .
$$




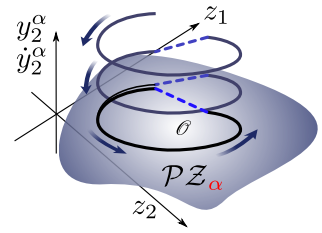

(a) Periodic Orbit

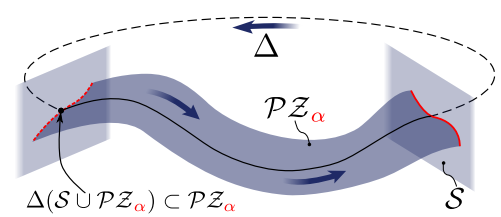

(b) Hybrid Invariance
Fig. 4: Illustration of the hybrid invariant periodic orbit on the partial hybrid zero dynamics (PHZD) surface. A PHZD surface is constructed via choosing the proper parameters set $\alpha$ through optimization.

Moreover, applying the feedback controllers to the hybrid control system given in (2) yields a hybrid system model, given as

$$
\mathscr{H}=(\mathscr{D}, S, \Delta, F) .
$$

with $\mathscr{U}=\emptyset$. In the next section, we introduce virtual constraints as a means to synthesize feedback controllers that realize stable walking of the human exoskeleton system.

\section{B. Partial Hybrid Zero Dynamics}

At the core of our controller approach is designing a set of virtual constraints that modulate the system behavior in order to achieve certain desired trajectories [7], [28]. Enforcing virtual constraints results in a reduced dimensional representation of the full order system that captures the natural dynamics of the robot.

Virtual Constraints. For the particular case of the human exoskeleton system with actuated ankle joints, we choose the actual outputs to be a combination of velocity regulating term $y_{1}^{a}$ and position modulating terms $y_{2}^{a}$. Specifically, the velocity regulating term is chosen to be the forward hip velocity with a constant desired velocity $v_{d}$, and position modulating outputs are chosen to represent the synchronized motion of the rest of the mechanical system. The virtual constraints are defined as the difference between the actual and desired outputs:

$$
\begin{aligned}
y_{1}(q, \dot{q}, \alpha) & =y_{1}^{a}(q, \dot{q})-v_{d}, \\
y_{2}(q, \alpha) & =y_{2}^{a}(q)-y_{2}^{d}(\tau(q), \alpha),
\end{aligned}
$$

where $y_{1}$ and $y_{2}$ are relative degree 1 and (vector) relative degree 2 by definition, $y_{2}^{d}$ is a vector of desired trajectories. In this paper, we use Bézier polynomials to represent the desired outputs with $\alpha$ being a set of Bézier coefficients, and $\tau(q)$ the state-based timing variable for synchronization [6].

Feedback Linearization. With the goal of driving the virtual constraints in (9) and (10) to zero exponentially, we use the following feedback control law,

$$
\begin{aligned}
u^{\alpha, \varepsilon}= & -\mathscr{A}^{-1}\left(\left[\begin{array}{c}
0 \\
L_{f}^{2} y_{2}(q, \dot{q}, \alpha)
\end{array}\right]\right. \\
& \left.+\left[\begin{array}{c}
L_{f} y_{1}(q, \dot{q}) \\
2 \varepsilon L_{f} y_{2}(q, \dot{q}, \alpha)
\end{array}\right]+\left[\begin{array}{c}
\varepsilon y_{1}(q, \dot{q}, \alpha) \\
\varepsilon^{2} y_{2}(q, \alpha)
\end{array}\right]\right),
\end{aligned}
$$

with a control gain $\varepsilon>0$ and decoupling matrix

$$
\mathscr{A}=\left[\begin{array}{c}
L_{g} y_{1}^{a}(q, \dot{q}) \\
L_{g} L_{f} y_{2}(q, \dot{q}, \alpha)
\end{array}\right],
$$

which is invertible due to the specific choice of virtual constraints. Applying this control law to (5) yields linear output dynamics of the form:

$$
\begin{aligned}
& \dot{y}_{1}=-\varepsilon y_{1}, \\
& \ddot{y}_{2}=-2 \varepsilon \dot{y}_{2}-\varepsilon^{2} y_{2} .
\end{aligned}
$$

Under the control law, the system in (5) renders an invariant reduced dimensional surface, termed as zero dynamics [28]:

$$
\begin{aligned}
\mathscr{Z}_{\alpha}=\{(q, \dot{q}) \in \mathscr{D} \mid & y_{1}(q, \dot{q}, \alpha)=0, \\
& \left.y_{2}(q, \alpha)=0, \dot{y}_{2}(q, \dot{q}, \alpha)=0\right\} .
\end{aligned}
$$

Since the velocity $\dot{q}$ undergoes a jump at impact (6), and $v_{d}$ is a constant, then the output $y_{1}$ necessarily undergoes a jump (see (9)), and the zero dynamics surface cannot be invariant through impact. Thus, we prefer to ignore this output and consider the following partial zero dynamics surface, given by:

$$
\mathscr{P} \mathscr{Z}_{\alpha}=\left\{(q, \dot{q}) \in \mathscr{D} \mid y_{2}(q, \alpha)=0, \dot{y}_{2}(q, \dot{q}, \alpha)=0\right\} .
$$

It is straightforward to show that the control law in (11) renders the partial zero dynamics submanifold invariant, i.e., any solution that starts in $\mathscr{P} \mathscr{Z}_{\alpha}$ remains in $\mathscr{P} \mathscr{Z}_{\alpha}$ until reaching a guard [7]. However, it is not necessarily invariant through impacts. To this end, the set of parameters $\alpha$ is constrained to be such that the partial zero dynamics is invariant through impacts. There exists a set of parameters $\alpha$ so that the submanifold $\mathscr{P} \mathscr{Z}_{\alpha}$ is impact invariant if

$$
\Delta\left(S \cap \mathscr{P}_{\mathscr{Z}_{\alpha}}\right) \in \mathscr{P} \mathscr{Z}_{\alpha}
$$

A manifold $\mathscr{P} \mathscr{Z}_{\alpha}$ is called hybrid invariant if it is invariant through impacts, and thus the hybrid system in (8) has a partial hybrid zero dynamics (PHZD), denoted as $\mathscr{H} \mid \mathscr{P} \mathscr{Z}_{\alpha}$. By enforcing partial hybrid zero dynamics, the full order dynamics of the hybrid system can be captured in a reduceddimension dynamical system that is independent of control inputs. Finding such parameters is typically formulated as a nonlinear constrained optimization problem [7], [17].

Theorem 1. If the hybrid system $\mathscr{H}$ in (8) satisfies (17), then there exists $\bar{\varepsilon}>0$ such that for all $\varepsilon>\bar{\varepsilon}$ the feedback controller (11) yields a periodic orbit $\mathscr{O}=\imath_{0}\left(\mathscr{O}_{P Z}\right)$, for $\mathscr{O}_{P Z} \subset \mathscr{P} \mathscr{Z}_{\alpha}$ and $\imath_{0}: \mathscr{P}_{\mathcal{Z}} \rightarrow \mathscr{D}$ the canonical embedding, that is a locally exponentially stable periodic orbit of $\mathscr{H}$.

Importantly, this theorem implies that the robot represented by the hybrid system $\mathscr{H}$ has an exponentially stable walking gait. Space constraints do not allow for a proof of this result, but it follows in a straightforward manner from the results of [7].

Remark 1. Introducing partial zero dynamics not only allows us to command a constant desired velocity, but also the evolution of $y_{1}$ is now solely determined via the linear output dynamics given in (13) and is independent of $\tau$. This allows for a driving element that pushes the robot forward regardless of the state of the phase variable. 


\section{Gait Generation And Simulations}

Using the PHZD framework described above, we want to design the desired profile of the virtual constraints that when enforced leads to a stable walking gait. This is done through a nonlinear constrained optimization process using direct collocation. Others ways of solving the optimization can be used like single shooting methods, but direct collocation was found to be the fastest and most efficient way to solve this problem [17]; stable walking gaits are obtained in minutes. In this section we presents an overview of how we used direct collocation to obtain stable dynamic walking gaits.

\section{A. Direct Collocation Optimization}

Direct collocation methods have been widely used for trajectory optimization of dynamical systems governed by ordinary differential equations [9], [16]. In this work, we incorporated the virtual constraints based feedback controller in the direct collocation based trajectory optimization problem. The PHZD constraint in (17) is enforced at impact, such that the optimized trajectory is a hybrid invariant periodic orbit. The end result is a set of parameters $\alpha$ for a stable walking gait.

The mathematical foundation of the direct collocation optimization is based on the finite step implicit Runge-Kutta methods [9]. Specifically, we divide the continuous dynamics solution into a fixed number of intervals. Let

$$
0=t_{0}<t_{1}<t_{2}<\cdots<t_{N}=T_{I},
$$

be the discrete time instances, a.k.a., nodes, defined at the terminal of these intervals, where $T_{I}>0$ is the time at which the swing foot hits the ground. Also, we introduce $x^{i}, \dot{x}^{i}, u^{i}$, and $F^{i}$ as decision variables of the optimization problem to approximate the state variables, control inputs, ground reaction forces of the system at node $i$, and introduce $\alpha^{*}$ as decision variables for the virtual constraints parameters, respectively. Next, we modify the classical HermiteSimpson method over time intervals between two neighboring even nodes. Specifically, the following equality constraintstermed as defect constraints-are enforced:

$$
\begin{aligned}
\dot{x}^{i}-3\left(x^{i+1}-x^{i-1}\right) / 2 \Delta t^{i}+\left(\dot{x}^{i-1}+\dot{x}^{i+1}\right) / 4 & =0, \\
x^{i}-\left(x^{i+1}+x^{i-1}\right) / 2-\Delta t^{i}\left(\dot{x}^{i-1}-\dot{x}^{i+1}\right) / 8 & =0,
\end{aligned}
$$

for all $i \in\{1,3, \cdots, N-1\}$, where $\Delta t^{i}=t_{i+1}-t_{i-1}$ is the time interval. If the Lagrangian dynamics in (3) and holonomic constraints in (4) are satisfied at each node, then the piecewise cubic polynomials that connect all $x^{i}$ represent an approximated solution of the differential equations in (5) [9], [17].

Considering that our goal is to find virtual constraints parameters instead of open-loop trajectories, we incorporate the feedback controller into the optimization in a way that is similar to holonomic constraints. Instead of enforcing the control input $u^{i}$ directly as in (11), we impose equality constraints on system states such that they satisfy the output dynamics in (13) and (14). Further, the hybrid invariant condition in (17) between states at the last and first nodes.
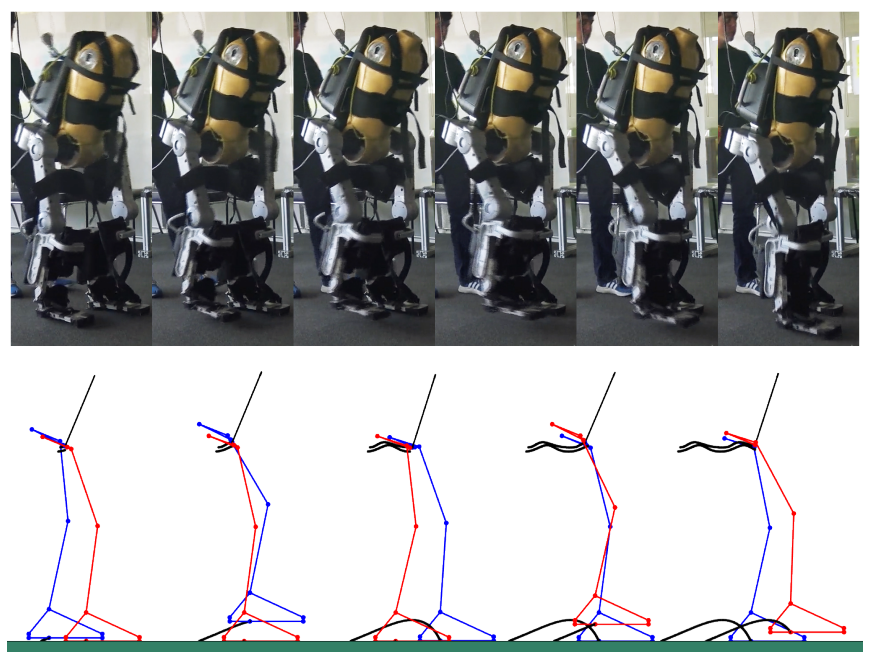

Fig. 5: Simulation of the gait for the exoskeleton with a dummy inside.

For detailed setup of the optimization problem, we refer the readers to [6].

In this work, we state the direct collocation based human exoskeleton gait optimization to minimize the mechanical cost of Transport (CoT), $\Phi_{C o T}$, of the gait, i.e.,

$$
\begin{aligned}
\underset{\mathbf{z}^{*}}{\operatorname{argmin}} & \Phi_{C o T}(\mathbf{z}) \\
\text { s.t } & \mathbf{z}_{\min } \leq \mathbf{z} \leq \mathbf{z}_{\max }, \\
& \mathbf{c}_{\min } \leq \mathbf{c}(\mathbf{z}) \leq \mathbf{c}_{\max },
\end{aligned}
$$

where $\mathbf{z}$ is the set of all decision variables, and $\mathbf{c}(\mathbf{z})$ is a collection of necessary constraints presented in [17]. Torque limits and joint position and velocity limits of the ATALANTE are directly enforced as boundary conditions of decision variables in (22), whereas friction cone and zero moment constraints of the foot contacts are enforced as extra physical constraints. In addition, several constraints are considered in the optimization in order to narrow down the search space and address certain aspects specific to humanfriendly walking. Impact velocities, ZMP position, CoM position and torso orientation are an example of the many constraints that need to be considered.

A challenge arises here, as each patient has a specific mass distribution and dimensions which lead to unique dynamics for each individual. In order to accelerate this process, Wandercraft has derived a fast way to recompute the dynamics for each case, making it possible to rapidly adapt to new patients. For now, the details of this process must be kept private to protect the intellectual property of the company. The result of this process (cf. Fig. 2) is a provably stable walking gait that minimizes a cost function (eg. energy consumption) and satisfies all of the desired constraints.

\section{B. Simulation results}

Even though the generated gaits are consistent with the dynamics by design, with collocation optimization, this only holds at the few specific node points selected for the virtual constraints. Therefore, in order to verify the validity of these 


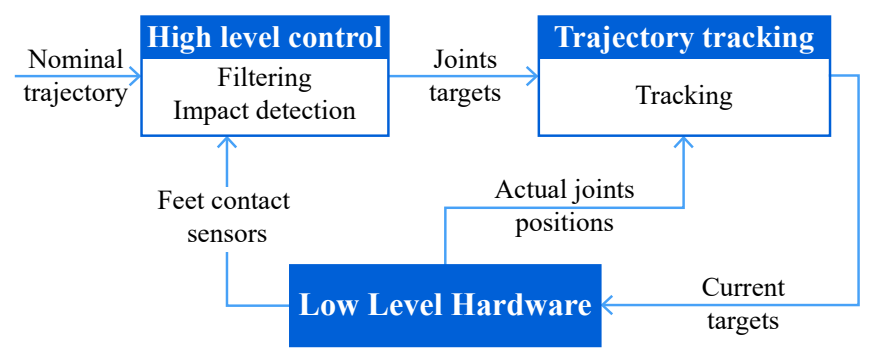

Fig. 6: Structure of the control loop.

gaits, a feedback-linearizing controller tracking the nominal outputs is implemented in a simulation. This way, the full trajectory of the robot is recovered and the periodicity and stability of the gait are assessed. The joint positions generated by these simulations are then used as the nominal joint trajectories to be tracked on the hardware. Gait tiles simulated walking gait obtained via this procedure is shown in Fig. 5 with the corresponding phase portrait plotted in Fig. 7 as the nominal gait (red), prior to modification of the trajectories on the hardware (shown in blue). The fact that the phase portraits are periodic orbits imply that a valid periodic walking gait is generated.

\section{IMPLEMENTATION ON THE EXOSKELETON}

The final step of this work is the implementation of the generated gaits on hardware. This section details some of the implementation on ATALANTE, and presents the results obtained with multiple users (1 dummy and 3 paraplegic patients). Importantly, dynamically stable locomotion is realized in all cases, and the walking is compared across patients.

\section{A. Implementation of gaits on hardware}

In order to translate the gaits obtained in simulation into actual dynamical walking on hardware, the following methodology has been followed (as illustrated in Fig. 2).

Adjusting the Exoskeleton dimensions. Since all patients have different sizes and body shapes, the hardware has been designed so that certain dimensions can be adjusted (cf. green links in Fig. 3). Using the measurements made to generate the patient model, the dimensions of the exoskeleton are adjusted to align the robot's joints with the patient's joints. This allows to minimize the internal stresses at the attachment points between the patient and the exoskeleton arising from the highly hyper-static nature of the humanexoskeleton mechanism.

Embedded systems structure. The exoskeleton is controlled by a central computer board running a real-time operating system and in charge of all high level computations. This central computer then sends commands to motor controllers that handle the low-level operation of brushless motors. Digital encoders are present on all the joints of the exoskeleton. IMU's are located in different parts of the exoskeleton allowing for a better estimation of the pose of the robot. Furthermore, sensors are present in the feet and provide a measurement of the center-of-pressure during walking.

In order to get the exoskeleton to realize the desired gait, trajectory tracking is implemented (cf. Fig. 6). Given
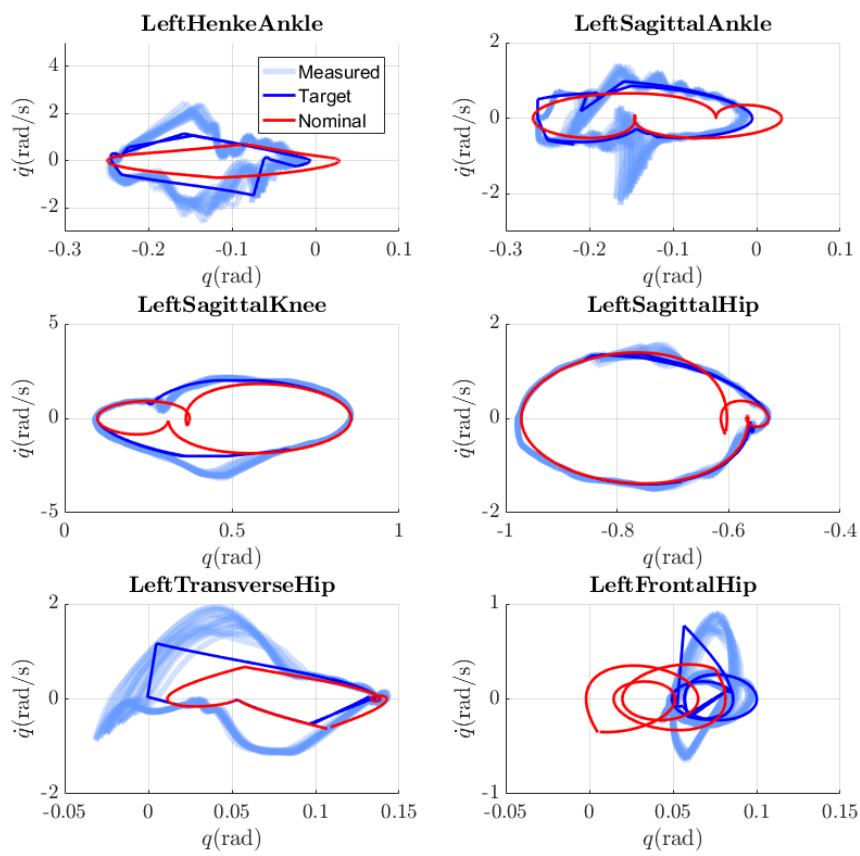

Fig. 7: Phase portraits for the dummy during 20s of unassisted walking.

one chosen cyclic gait, articular targets for each joints are generated. These targets are the nominal trajectories of the joints but modified online based on foot impact detection to allow for a better transition between steps. These targets are then sent to a control loop which generates current commands at the joint level. The current command is then sent to the motor controller that handles the high-frequency low-level control of the motor.

Tuning of the trajectory. Finally, the nominal trajectory is tuned in order to accommodate for the mechanical imperfections and asymmetries of the robot. This tuning happens during a series of walking experiments with a mannequin until the desired behavior is achieved. Tuning generally improves the stability and robustness of the gait after a few trials.

\section{B. Experimental results with a dummy}

To evaluate the controllers, experiments are first carried out using a mannequin or dummy (cf. Fig. 5). As we can see in Fig. 7, the nominal and target trajectories (in red and blue respectively) are marginally different after the tuning and high-level filtering of the nominal trajectories (cf. Fig. 6). The target gait is followed with relatively good accuracy resulting in stable dynamic walking of the hardware.

\section{Experimental results with human subjects}

As a result of the successful results obtained with the dummy, experiments are carried out with paraplegic patients. Some characteristics of these patients are summarized in Table I.

Clinical Set-Up. Experiments were conducted in a certified medical center and approved by the ANSM (French regulatory administration for health products). To prevent 
TABLE I: Patients data

\begin{tabular}{c|c|c|c|c} 
Patient & Height(m) & Weight(kg) & $\begin{array}{c}\text { Distance } \\
\text { traveled(m) }\end{array}$ & speed(m/s) \\
\hline A & 1.80 & 68 & 8.9 & 0.11 \\
B & 1.69 & 80 & 10.56 & 0.15 \\
C & 1.80 & 75 & 9.5 & 0.13
\end{tabular}
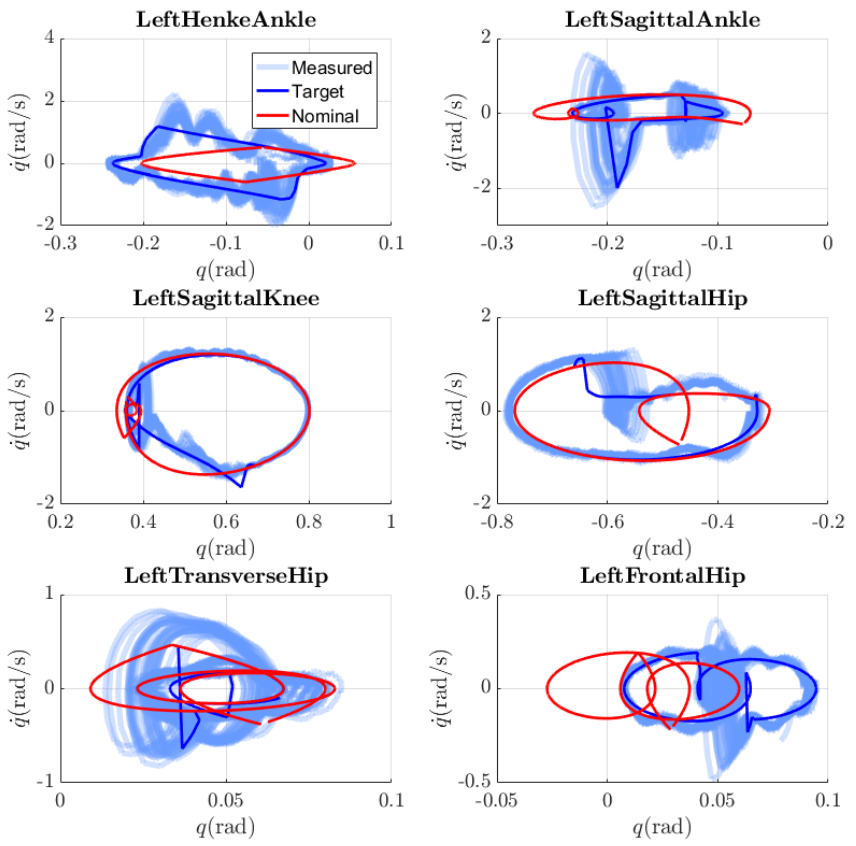

Fig. 8: Phase portraits for patient A during 60s of unassisted walking.

injury from a fall, one person is placed at each side of a patient. In case of loss of balance, the two assistants catch the exoskeleton by handles on its sides. Furthermore, a safety cable is attached to the exoskeleton and an overhead rail (or gantry). This is a secondary means to secure a patient and prevent a fall. Hence, assistance is provided only in case of loss of balance. During walking, the exoskeleton and its user are self stabilized and no outside assistance was given.

Results. As can be seen in the video at [4] and Fig. 9c, crutch-less dynamically stable exoskeleton walking of paraplegic patients is achieved as a result of the methodology developed in this paper. All patients managed to walk unassisted for the entire length of the room after a few trials during which a best gait was chosen and then tuned; Table I includes the speed of walking and distance traveled.

The ability to successfully transfer the formal gaits generated to hardware is illustrated in Fig. 8 for Patient A, wherein the nominal (blue) and measured (shaded) trajectories are consistent throughout the experiment. The motors torques resulting from tracking the nominal trajectories (cf. Fig. 9b) are also consistent with simulation. Note that motor-torque saturations are relatively uncommon as the gaits are designed to account for all hardware limits. To compare the walking between patients, a representative selection of phase portraits for each patient are presented in Fig. 9a; even though the gaits are not the same (as they have been generated to best suit each patient), they all display a common fundamental structure.

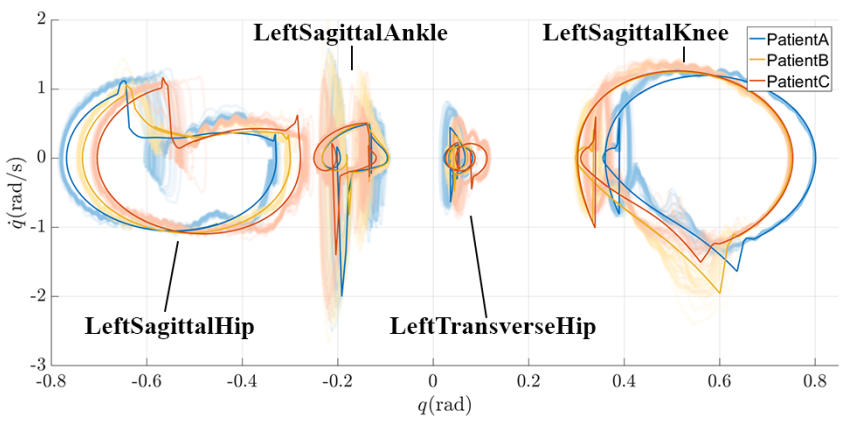

(a) Selection of phase portraits. The solid lines are the target trajectories. The shaded regions are the measured joints positions.

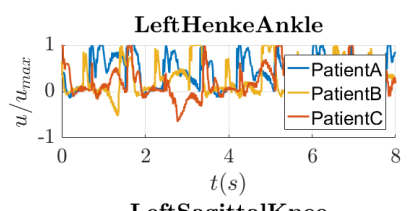

LeftSagittalKnee

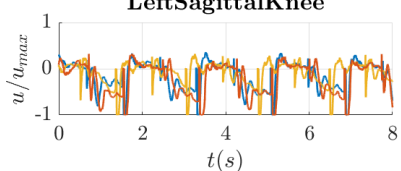

LeftTransverseHip

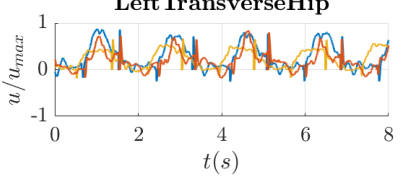

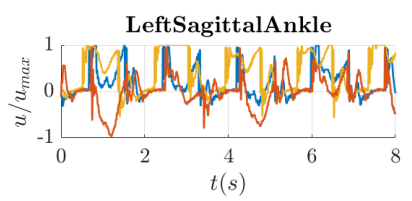

LeftSagittalHip

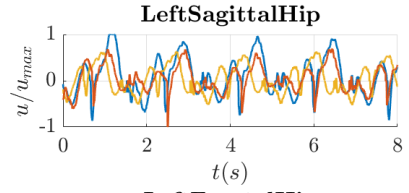

LeftFrontalHip

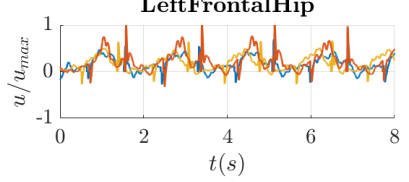

$t(s$ (b) Measured motor torques.
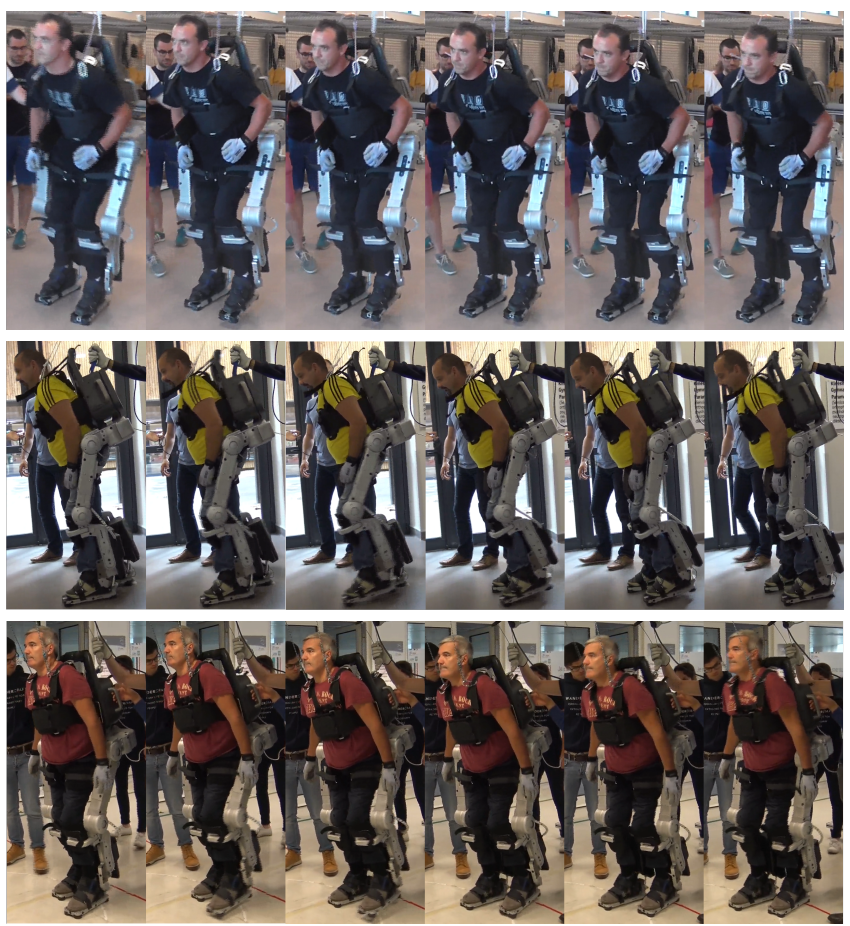

(c) Walking tiles of patients A, B and C (in this order from top to bottom).

Fig. 9: Experimental results for all three patients. 
This is further illustrated in gait tiles of the patients walking in the exoskeleton (cf. Fig. 9c). While the dynamic walking gaits obtained are preliminary, and in no way constitute any kind of clinical evaluation, the ability to consistently realize them on patients points toward the validity of the framework developed.

\section{Conclusions}

This paper has demonstrated the first ever experimental realization of hands-free dynamic walking by subjects with a lower spine injury. The keys to realizing this were novel hardware, stiff enough to physically support a subject, powerful enough to move the device's legs quickly, and novel control mathematics developed over the past 15 years to allow bipedal robots to walk stably in uncertain environments and with imprecise dynamic models. Importantly, these results concretely mark the first steps toward the vision of restoring locomotion for paraplegics.

The preliminary results demonstrated very slow walking on the order of $0.1 \mathrm{~m} / \mathrm{s}$. Stable gaits at $0.4 \mathrm{~m} / \mathrm{s}$ were achieved in simulation, and such speeds can be expected to be reached on the current hardware and with patients. Future work will also provide the ability to stand from a chair, initiate a gait, stop, turn, and sit in a chair. However, before providing more gait features, focus will be on improving the basic posture of the gaits to be more upright and comfortable, with foot roll and heel strike. Better robustness of the overall closedloop system is also a general goal. The hope is that these future research directions will enable dynamic exoskeleton locomotion in everyday life settings, from traversing up and down stairs to walking outdoors in natural environments.

\section{ACKNOWLEDGMENT}

The authors would like to thank the entire Wandercraft team which designed ATALANTE, implemented and tested the control algorithms both in simulation and in real conditions. Additionally, the authors are grateful to Laurent Praly and Nicolas Petit, researchers at CAS (Mines ParisTech, PSL Research University) for their precious scientific support since the beginning of Wandercraft, and to Koushil Sreenath at UC Berkeley for collaborative efforts on realizing dynamic walking on exoskeletons.

\section{REFERENCES}

[1] Ekso bionics. http://eksobionics.com/eksohealth/patients/. Accessed: 2017-09-15.

[2] Rewalk. http://rewalk.com/. Accessed: 2017-09-15.

[3] Rex bionics. http://www.rexbionics.com/. Accessed: 2017-09-15.

[4] Video of the experimental results. https://youtu.be/V30HsyUD4fs.

[5] Wandercraft. http://www.wandercraft.eu/. Accessed: 2017-09-15.

[6] Ayush Agrawal, Omar Harib, Ayonga Hereid, Sylvain Finet, Matthieu Masselin, Laurent Praly, Aaron D. Ames, Koushil Sreenath, and Jessy W. Grizzle. First steps towards translating HZD control of bipedal robots to decentralized control of exoskeletons. IEEE Access, 5:99199934, 2017.

[7] Aaron D Ames. Human-inspired control of bipedal walking robots. IEEE Transactions on Automatic Control, 59(5):1115-1130, 2014.

[8] Aaron D Ames, Kevin Galloway, Koushil Sreenath, and Jessy W Grizzle. Rapidly exponentially stabilizing control lyapunov functions and hybrid zero dynamics. IEEE Trans. on Automatic Control, 59(4):876-891, 2014.
[9] John T Betts. Survey of numerical methods for trajectory optimization Journal of guidance, control, and dynamics, 21(2):193-207, 1998.

[10] Aaron M. Dollar and Hugh Herr. Lower extremity exoskeletons and active orthoses: Challenges and state-of-the-art. IEEE Trans. Robot., 24(1):144-158, 2008

[11] Kevin Fite, Jason Mitchell, Frank Sup, and Michael Goldfarb. Design and control of an electrically powered knee prosthesis. In Rehabilitation robotics, 2007. ICORR 2007. IEEE 10th international conference on, pages 902-905. IEEE, 2007.

[12] Kevin Galloway, Koushil Sreenath, Aaron D. Ames, and Jessy W. Grizzle. Torque saturation in bipedal robotic walking through control lyapunov function based quadratic programs. IEEE Access, 3:323-332, April 2015.

[13] Brent Griffin and Jessy Grizzle. Walking gait optimization for accommodation of unknown terrain height variations. In 2015 American Control Conference, pages 4810-4817, 2015.

[14] J. W. Grizzle, C. Chevallereau, R. W. Sinnet, and A. D. Ames. Models, feedback control, and open problems of 3D bipedal robotic walking. Automatica, 50(8):1955 - 1988, 2014.

[15] J Grundmann and A Seireg. Computer control of multi-task exoskeleton for paraplegics. In Proceedings of the Second CISM/IFTOMM International Symposium on the Theory and Practice of Robots and Manipulators, pages 233-240, 1977.

[16] Charles R Hargraves and Stephen W Paris. Direct trajectory optimization using nonlinear programming and collocation. Journal of Guidance, Control, and Dynamics, 10(4):338-342, 1987.

[17] Ayonga Hereid, Eric A Cousineau, Christian M Hubicki, and Aaron D Ames. 3D dynamic walking with underactuated humanoid robots: A direct collocation framework for optimizing hybrid zero dynamics. In Robotics and Automation (ICRA), 2016 IEEE International Conference on, pages 1447-1454. IEEE, 2016.

[18] D Hristic, M Vukobratovic, and M Timotijevic. New model of autonomous' active suit'for distrophic patients. In Proceedings of the International Symposium on External Control of Human Extremities, pages 33-42, 1981.

[19] Y. Hurmuzlu, F. Génot, and B. Brogliato. Modeling, stability and control of biped robots-a general framework. Automatica, 40(10):16471664, 2004.

[20] H Igo Krebs, Neville Hogan, Mindy L Aisen, and Bruce T Volpe. Robot-aided neurorehabilitation. IEEE transactions on rehabilitation engineering, 6(1):75-87, 1998.

[21] R. M. Murray, Z. Li, S. S. Sastry, and S. S. Sastry. A mathematical introduction to robotic manipulation. CRC press, 1994.

[22] Jacob Reher, Eric A Cousineau, Ayonga Hereid, Christian M Hubicki, and Aaron D Ames. Realizing dynamic and efficient bipedal locomotion on the humanoid robot durus. In Robotics and Automation (ICRA), 2016 IEEE International Conference on, pages 1794-1801. IEEE, 2016

[23] Jacob P Reher, Ayonga Hereid, Shishir Kolathaya, Christian M Hubicki, and Aaron D Ames. Algorithmic foundations of realizing multi-contact locomotion on the humanoid robot durus. In Twelfth International Workshop on Algorithmic Foundations on Robotics, 2016.

[24] A Seireg and JG Grundmann. Design of a multitask exoskeletal walking device for paraplegics. Biomechanics of Medical Devices, pages 569-644, 1981

[25] Frank Sup, Amit Bohara, and Michael Goldfarb. Design and control of a powered transfemoral prosthesis. The International journal of robotics research, 27(2):263-273, 2008.

[26] M Vukobratovic, D Hristic, and Z Stojilikovic. Development of active anthropomorphic exoskeletons. Medical and Biological Engineering, 12(1):66-80, 1974.

[27] Michael Wehner, Brendan Quinlivan, Patrick M Aubin, Ernesto Martinez-Villalpando, Michael Baumann, Leia Stirling, Kenneth Holt, Robert Wood, and Conor Walsh. A lightweight soft exosuit for gait assistance. In Robotics and Automation (ICRA), 2013 IEEE International Conference on, pages 3362-3369. IEEE, 2013.

[28] E. R. Westervelt, J. W. Grizzle, C. Chevallereau, J. H. Choi, and B. Morris. Feedback control of dynamic bipedal robot locomotion. CRC press Boca Raton, 2007.

[29] Juanjuan Zhang, Chien Chern Cheah, and Steven H Collins. Experimental comparison of torque control methods on an ankle exoskeleton during human walking. In Robotics and Automation (ICRA), 2015 IEEE International Conference on, pages 5584-5589. IEEE, 2015. 